\title{
Matérián
}

ISSN 1517-7076

\section{Desenvolvimento das Relações Interfalangeanas e metacarpo-falangeanas para os Dedos Durante Movimentos de Pinças}

\author{
Danilo A. P. Nagem ${ }^{1}$, Marco A. G. Moreira ${ }^{3}$, Guilherme A. S. Pereira ${ }^{3}$, \\ Carlos J. Tierra-Criollo ${ }^{3}$, Marcos B. Pinotti ${ }^{2}$ \\ ${ }^{1}$ Programa de Pós-Graduação em Engenharia Mecânica, Universidade Federal de Minas Gerais, \\ DEMEC/UFMG, CEP 31270-901, Belo Horizonte, M. G., Brasil \\ e-mail: dapin@ufmg.br \\ ${ }^{2}$ Departamento de Engenharia Mecânica, UFMG \\ e-mail:pinotti@demec.ufmg.br \\ ${ }^{3}$ Departamento de Engenharia Elétrica, UFMG \\ e-mail: lelinhosgp@yahoo.com.br, gpereira@cpdee.ufmg.br, carjulio@cpdee.ufmg.br
}

\begin{abstract}
RESUMO
Este trabalho determina as relações entre os ângulos das articulações interfalangeanas e metacarpofalangeanas para os dedos indicador, médio, anular e mínimo da mão humana durante alguns movimentos de pinça com o objetivo de desenvolver uma prótese multifuncional de mão com movimentos antropomórficos. Uma das grandes dificuldades encontradas para o desenvolvimento destas relações é o fato da mão apresentar um elevado número de graus de liberdade, dificultando a análise dos dados. Utiliza-se um sistema de captura de imagem em apenas um plano para analisar o movimento. Determinam-se as relações para os movimentos de flexão e extensão dos dedos, desprezando adução e abdução durante a realização destas pinças.
\end{abstract}

Palavras chaves: Mão, movimentação, captura de imagens, relações, ângulos.

\section{Development of the Relations for the Interphalange and metacharpo-phalange During Grasp Movement}

\section{ABSTRACT}

This works presents the relation for the angles of interphalanges and metacharpo-phalange joints for the index, middle, ring and little finger of human hand. These angles are determinated during a grasp movement. These relations will be used to develop a hand prosthesis with anthropomorphic movements. The main difficult found in this work was the highest numbers of degrees of freedom from the human hand. A methodology was developed to find these angles. This methodology analyzed the movement in 2-D space, and use only one digital camera and a computer. This methodology could be used for determinate these relations for prosthesis because the abduction/adduction movements was almost never applied in this kind of mechanism.

Keywords: Hand, movement, image capture, angle relation.

\section{INTRODUÇÃO}

A mão humana apresenta para cada um dos dedos quatro graus de liberdade, com exceção do polegar, que apresenta cinco, sendo três de flexão e extensão e uma de adução e abdução, ainda dois graus de liberdade para o punho. Para toda esta liberdade de movimentação a mão possui 27 (vinte e sete) ossos e utiliza inúmeros músculos intrínsecos e extrínsecos, inúmeros tendões e ligamentos. Uma estrutura que possua todas as características da mão seja dimensional, sensorial ou dinâmica, completamente semelhante à mão humana, ainda é um desafio para a ciência. Mãos robóticas [1-4] são as que apresentam uma maior semelhança dimensional e dinâmica com a mão humana, porém estes mecanismos apresentam grandes sistemas de controle. O que torna inviável seu uso em aplicações com intenções de restaurar o movimento humano. 
A alteração da movimentação da mão humana pode estar ligada a inúmeras patologias, sendo que quanto mais tardia a identificação correta, mais difícil pode ser o tratamento. Muitas vezes sendo necessário a utilização de uma órtese para restaurar o movimento ou até mesmo uma prótese para substituir o membro perdido.

Desde que o homem começou a desenvolver próteses de membros superiores, a primeira é datada de mais de 200 a.C. [్]], tem-se buscado recuperar as principais características deste membro. Dentre as várias funções que a mão apresenta, pode-se destacar a função de transmitir sensações, de expressar, de segurar, conduzir e de manipular objetos de diferentes formas.

Estudos recentes [므료 destacam a importância mecânica não apenas da capacidade de preensão dos objetos mas também como é realizado o este movimento. A maneira como este movimento é realizado é de grande importância para o usuário da prótese [8]. Com isto, os projetos de próteses mais recentes assemelham-se tanto fisicamente como dinamicamente com a mão humana, movimentos antropomórficos [911]. Obtêm-se uma maior adaptação por parte do paciente. Com isto têm-se dedicado à melhoria do projeto dos dedos, aplicando a estes características de movimentos mais antropomórficos.

Vários métodos estão sendo adotados para identificar estes movimentos. Os principais envolvem análise tridimensional através de captura de movimento [12, 13], outros envolvem captura de imagens utilizando ressonância magnética [14] para identificar o centro de rotação. Estes métodos apresentam uma alta qualidade de captura, mas um custo elevado. Outros métodos como o desenvolvido em [15], apresenta um baixo custo em relação aos métodos tridimensionais, mas só é capaz de analisar o movimento do dedo indicador e médio.

Este texto apresenta uma metodologia de baixo custo, para a determinação destas relações entre a movimentação das articulações dos dedos indicador, médio, anelar e mínimo. Sendo analisados os movimentos de flexão e extensão das articulações interfalangeanas [16] e metacarpo-falangeanas (MF).

\section{OBJETIVOS}

Este trabalho apresenta a metodologia desenvolvida para determinar as relações de extensão e flexão das articulações IF e MF dos dedos da mão, com exceção do polegar, utilizando um sistema de captura de imagens. Deve-se ser capaz de detectar uma alteração patológica ou até mesmo a melhora após um tratamento, quando comparada com uma medida anterior. Ela também deve permitir gerar as equações que relacionam os ângulos das articulações MF, com as articulações IF do mesmo dedo.

No desenvolvimento desta metodologia se enfatiza o baixo custo dos equipamentos e testes realizados.

\section{METODOLOGIA}

Durante o movimento da mão humana pode se observar uma relação entre os movimentos das falanges com a movimentação da articulação MF e até mesmo com o próprio movimento do pulso. Em muitos casos pode se observar uma relação entre o movimento de um dedo com outro. Isto pode ser observado, por exemplo, quando se posiciona a falange proximal em $90^{\circ}$ em relação ao metacarpo [17], não se consegue estender o dedo anelar sem gerar a movimentação dos outros dedos.

Por isto deve-se utilizar o movimento completo durante as pinças sem travar nenhum dos dedos, pois isto pode alterar a relação de movimentação das articulações. O movimento do pulso, também interfere nesta movimentação, sendo assim é necessário travar sua articulação em posição neutra para a geração do movimento. Esta medida é entre $14^{\circ}$ e $20^{\circ}$ entre a palma da mão e a ulna [17].

Para se travar o pulso do paciente foi desenvolvido uma órtese, figura 01, que se fixa ao antebraço do paciente estabilizando a articulação do punho em 20 graus de extensão e 14 graus de desvio ulnar. 


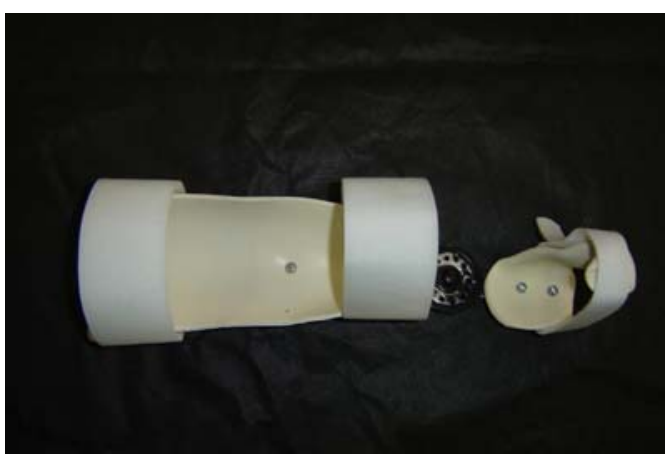

(a)

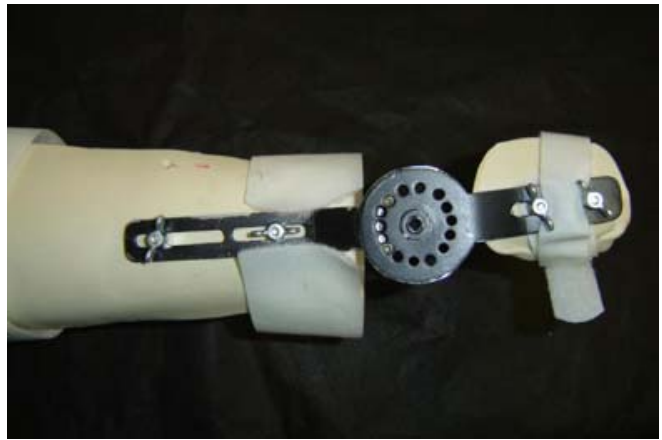

(b)

Figura 01: (a) Órtese de mão, (b) Detalhe que permite regular a inclinação no braço do punho do paciente.

Após a fixação do punho é preso ao dedo, a ser estudado, dois anéis com uma haste de movimentação com dois clusters cada (Fig. 2a) para a captura e processamento da imagem (Fig 2b). Assim como sobre a mão do paciente é colado um cluster no metacarpo para a identificação do ponto que representa o mesmo.

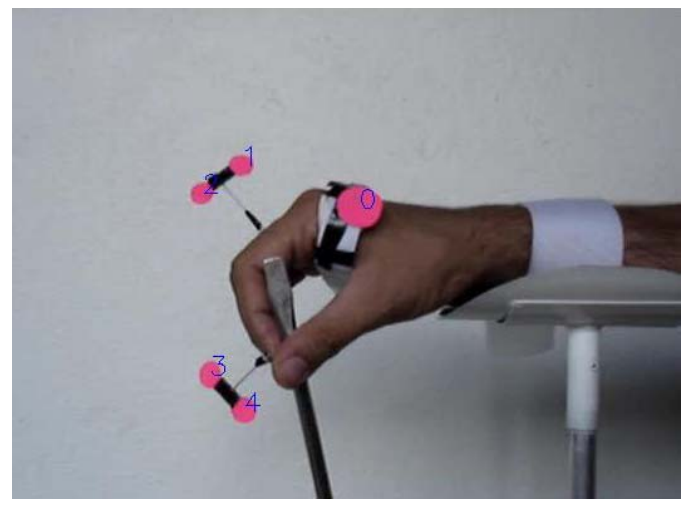

(a)

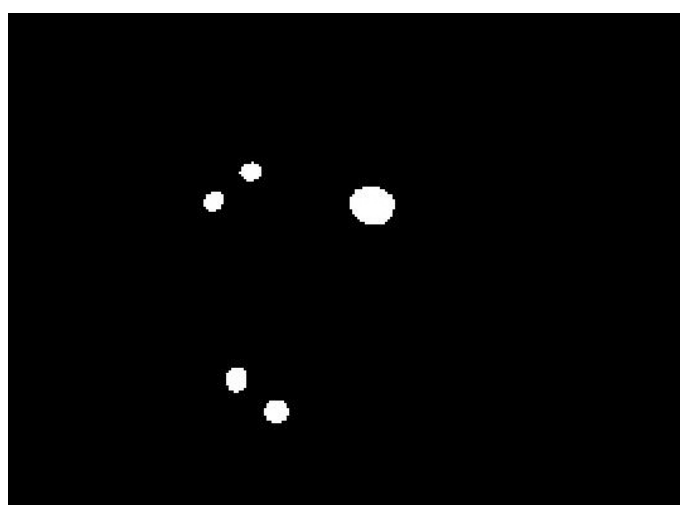

(b)

Figura 02: (a)Mão com Clusters, (b) Vídeo binarizado para analise.

Apóia-se o antebraço do paciente sobre um suporte para impedir o movimento. Posiciona-se a câmera de forma que o centro da imagem esteja alinhado com o cluster do centro do metacarpo para minimizar os erros de paralaxe. É então determinado ao paciente manter somente o dedo esticado para se fazer uma foto (Fig. 3), que serve como parâmetro para calibração do sistema. Após esta calibração o paciente realiza repetidos movimentos. Estes movimentos podem ser de pinça lateral, pinça polpa-a-polpa, pinça tri-digital, garra entre outros.

Como não ocorre movimento relativo entre o centro da articulação MF, IF proximal e a primeira haste com os clusters, utilizam-se as fotos para se determinar à posição relativa entre os eixos de rotação das articulações e o sistema de coordenadas geradas pelos dois clusters. É realizado o mesmo procedimento para se determinar às posições relativas da ponta do dedo e da articulação IF distal.

Com isto, através das relações trigonométricas consegue-se determinar $(\mathrm{X}, \mathrm{Y})$ do centro de rotação de cada articulação, como pode ser observado na figura 03, e nas equação 01 e 02. 


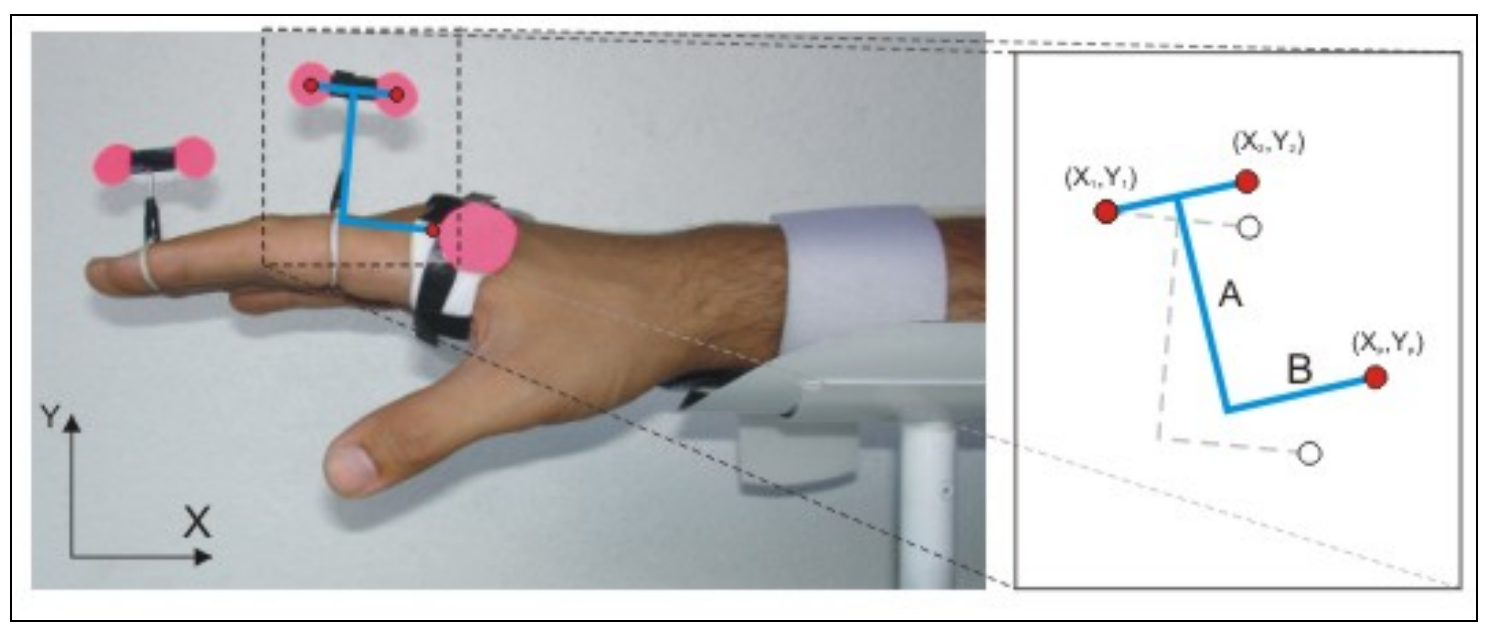

Figura 03: Sistema para auxilio da determinação do centro de rotação de cada articulação

$$
\begin{aligned}
& x_{p}=\frac{x_{1}+x_{2}}{2}+\frac{a\left(y_{2}-y_{1}\right)+b\left(x_{2}-x_{1}\right)}{\sqrt{\left(x_{2}-x_{1}\right)^{2}+}\left(y_{2}-y_{1}\right)^{2}} \\
& y_{p}=\frac{y_{1}+y_{2}}{2}+\frac{b\left(y_{2}-y_{1}\right)-a\left(x_{2}-x_{1}\right)}{\sqrt{\left(x_{2}-x_{1}\right)^{2}+}\left(y_{2}-y_{1}\right)^{2}}
\end{aligned}
$$

Foi desenvolvido um software capaz de identificar estes clusters e então determinar a posição de cada articulação assim como o ângulo entre cada segmento do dedo estudado. O movimento repetido pelo paciente ocorre em intervalos de tempos diferentes, por isto é necessário à normalização destes tempos para cada uma das repetições realizadas. Sendo então possível determinar uma média entre estes valores e a relação entre cada ângulo durante o movimento, determinando assim as equações para cada dedo.

\section{RESULTADOS E DISCUSSÃO}

Os dados coletados permitiram a determinação dos ângulos das articulações MCP, IFD e IFP para os dedos indicador, médio, anular e mínimo, para a mão de um paciente durante um movimento estabelecido. Movimento de pinça lateral. (Fig 04 (a)). Esta pinça de grande força é uma das principais pinças realizadas pelo ser humano.

Seu movimento completo é um dos mais complicados para ser determinado pelos métodos clássicos, de análise tridimensional, pois ocorre sempre a superposição ou ocultação de marcas.

Com a metodologia desenvolvida foi possível determinar a angulação das articulações durante todo o movimento. Este ângulo é determinado em relação à parte palmar da mão, como pode ser observado em relação à figura 04 (b). 


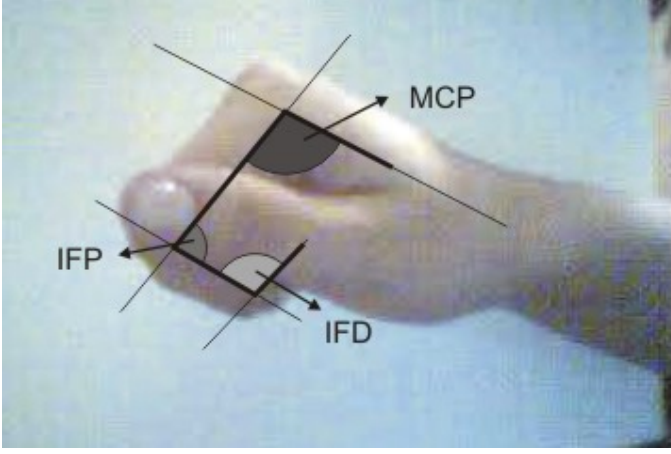

(a)

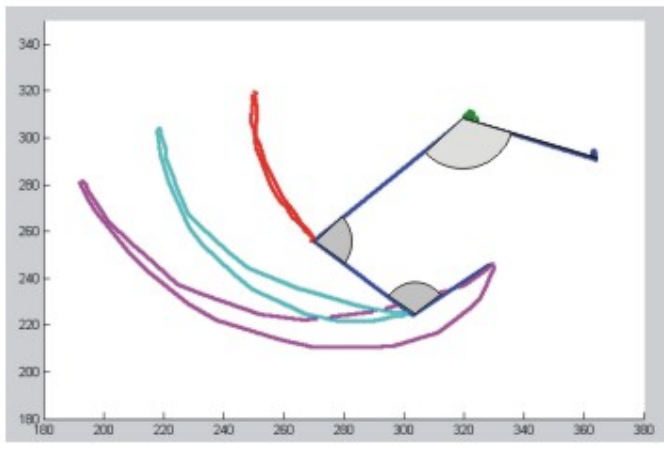

(b)

Figura 04: (a) Mão em posição de pinça lateral, (b) Determinação dos ângulos e trajetória dos dedos.

Devido à normalização dos tempos, os resultados obtidos das variações angulares são apresentados em relação a uma variável $x$ qualquer. Sendo que o tempo de realização do movimento $(t)$ é uma função linear de $x$. Este valor linear depende da velocidade que se deseja imprimir ao movimento.

Os valores dos ângulos podem ser observados nos gráficos das figuras 05 a 08 . As equações destes ângulos de cada articulação para cada um dos dedos são determinadas realizado um ajuste polinomial pelo método dos mínimos quadrados [15], como pode ser observado nas figuras correspondentes a seguir.

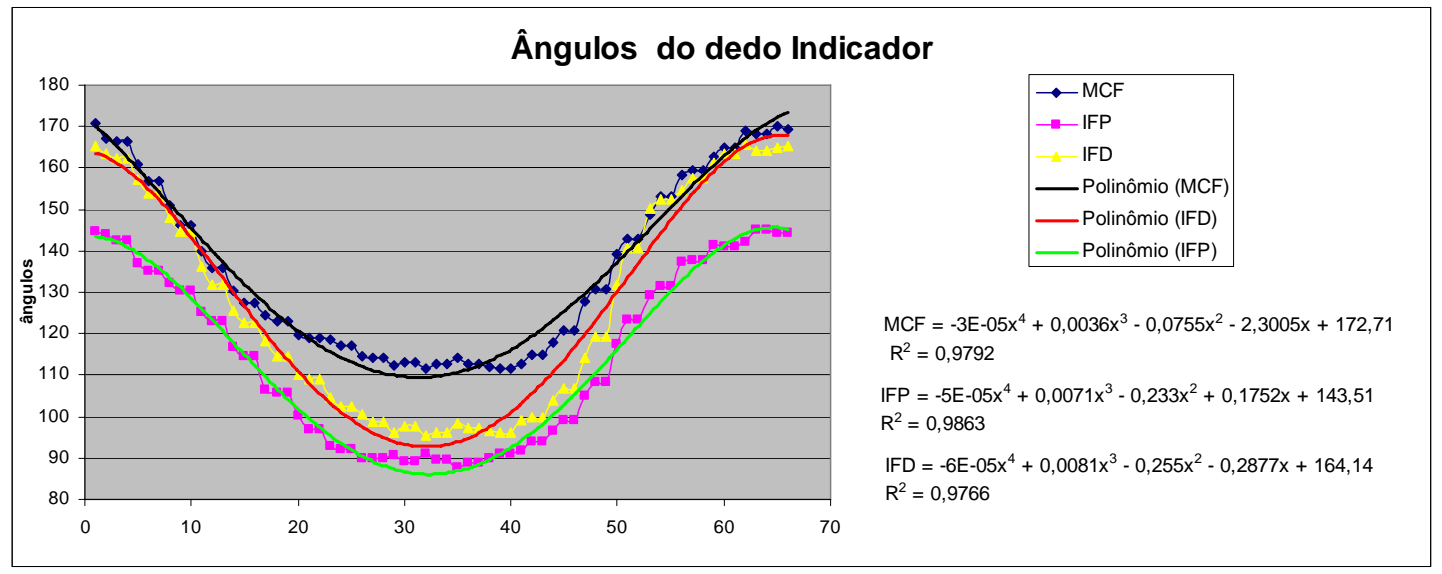

Figura 05: Representação gráfica e representação polinomial da angulação articular do dedo indicador.

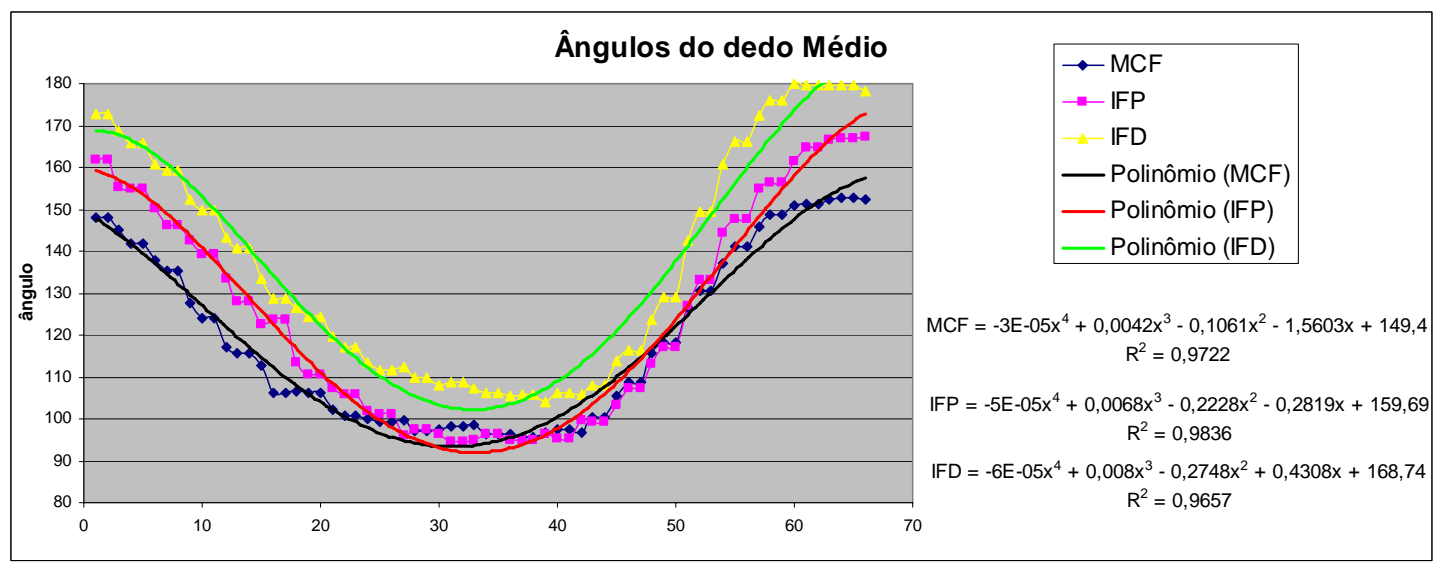

Figura 06: Representação gráfica e representação polinomial da angulação articular do dedo médio. 


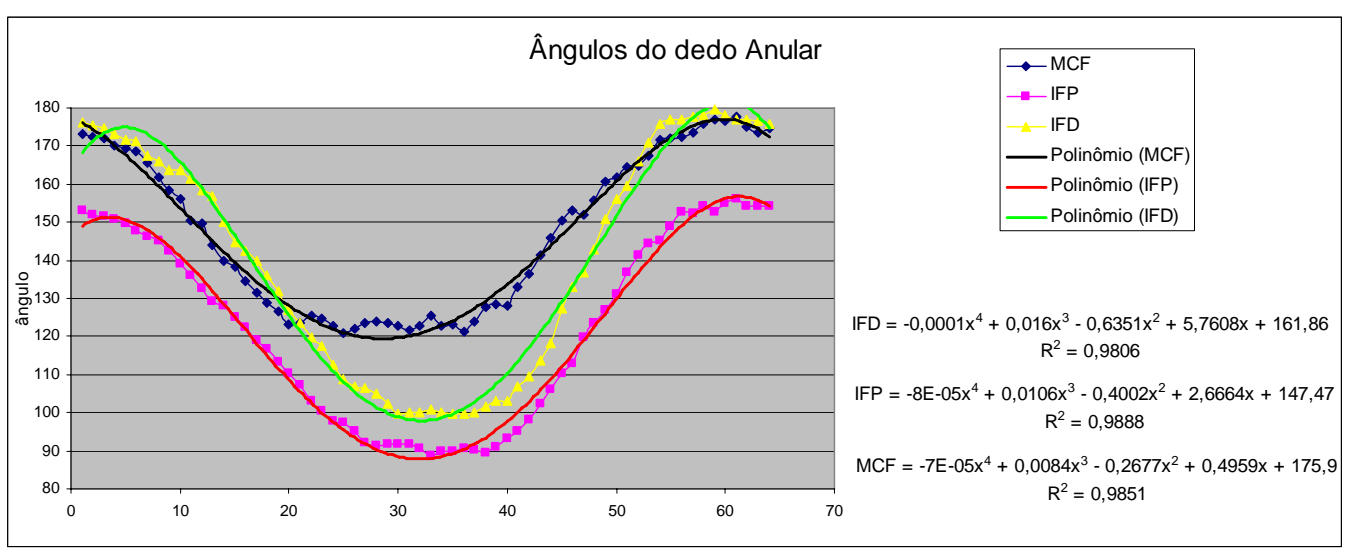

Figura 07: Representação gráfica e representação polinomial da angulação articular do dedo Anular.

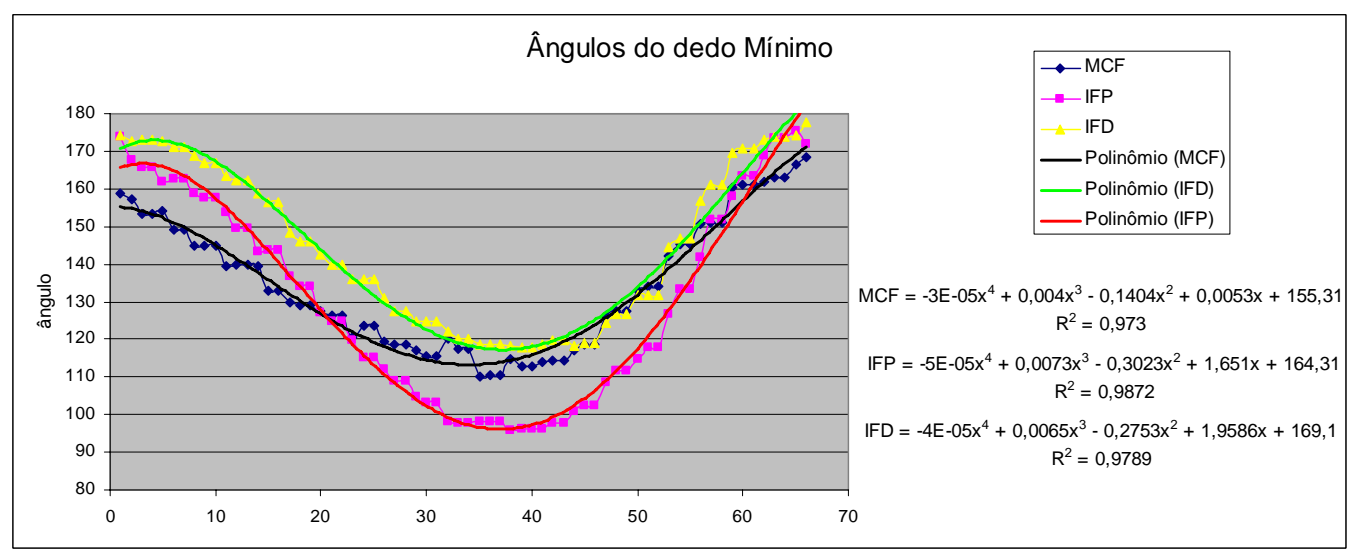

Figura 08: Representação gráfica e representação polinomial da angulação articular do dedo mínimo.

Com a metodologia desenvolvida foi possível determinar as equações para os ângulos de cada articulação dos dedos indicador, médio, anular e mínimo para um movimento de pinça lateral.

\section{CONCLUSÃO}

Com a metodologia proposta é possível determinar as equações entre as articulações interfalangeanas e metacarpo-falangeana para os dedos indicador, médio, anelar e mínimo para os movimentos de flexão e extensão. O método apresentou baixo custo em relação aos sistemas tradicionais de captura de imagem tri-dimensonal, pois utiliza uma câmera digital e um computador para a análise da imagem. Podendo assim auxiliar em diagnóstico de patologias e determinar a evolução de um tratamento fisioterápico ou de uma cirurgia.

Da posse destas equações é possível também controlar um movimento de uma prótese, desenvolvendo um mecanismo capaz de gerar para cada articulação o ângulo descrito. Sendo assim a prótese irá apresentar um movimento semelhante à mão humana.

Todas as equações deverão ser numeradas seqüencialmente.

\section{BIBLIOGRAFIA}

[1] DIFFLER, M.A., CULBERT, C.J., AMBROSE, R.O., PLATT, R.Jr., BLUETHMANN, W.J., "Evolution of the NASA/DARPA Robonaut Control System”, In: Conference on Robotics and Automation. Proceddigings.ICRA 03 IEEE International 2, pp. 2543-2548, 2003.

[2] KYBERD, P.J., LIGHT, C., CHAPPELL, P.H., NIGHTINGALE, J.M., WHATLEY, D., EVANS, M., "The Design of Anthropomorphic Prosthetic Hands: A Study of the Southampton Hand", Robotica, v. 19, pp. 593-600, 2001.

[3] LOTTI, F., TIEZZI, P., VASSURA, G., BIAGIOTTI, L., MELCHIORRI, C., "UBH 3: An Anthropomorphic Hand with Simplified Endo-skeletal Structure and Soft Continuous Fingerpads”, 
In: IEEE International Conference on Robotics and Automation. ICRA 04.2004 5, Italy, pp. 47364741, 2004.

[4] PONS, J.L., ROCON, E., CERES, R., REYNAERTS, D., SARO, B., LEVIN, S., VAN MOORLEGHEM, W., "The MANUS-HAND Dextrous Robotics Upper Limb Prosthesis: Mechanical and MAnipulation Aspects”, Autonomous Robots, v. 16, pp. 143-163, 2004.

[5] CARVALHO, D.C.L., SENE, M.,O., CUNHA, F.L., CLIQUET JR, A., "Adaptação dos Pacientes ao uso de Próteses Mioelétricas: Estudo do Tratamento Fisioterápico e Possíveis Alterações Fisiológicas", In: Congresso Iberoamericano Iberdiscap 2000, 3o de Comunicação Alternativa e Aumentatica e 10 de Tecnologias de Apoio Para Deficiência. IBERDISCAP., pp. 107-110, 2000.

[6] KYBERD, P.J., LIGHT, C., CHAPPELL, P.H., NIGHTINGALE, J.M., WHATLEY, D., EVANS, M., "The design of Anthropomorphic Prosthetic Hands: A Study of the Southampton Hand", Robotica, v. 19, pp. 593-600, 2001.

[7] LIGHT, C.M., CHAPPELL, P.H., "Development of a Lightweight and Adaptable multiple-axis Hand Prosthesis”, Medical Engineering \& Physycs, v. 22, pp. 679-684, 2001.

[8] CUNHA, F.L., SCHNEEBELI, H.A., DYNNIKOV, V.I., "Development of Anthropomorphic Upper Limb Prostheses with human-like Interphalangian and Interdigital Couplings”, Artificial Organs, v. 24, pp. 193-197, 2000.

[9] CAROZZA, M.C., MASSA, B., ZECCA, M., DARIO, P., “A 'Wearable’ Artificial Hand for Prosthetics and Humanoid Robotics Applications". In: Procedings of the 2001 IEEE - RAS International Conference on Humanoid Robots, 2001.

[10] CAROZZA, M.C., MASSA, B., MICERA, S., LAZZARINI, R., ZECCA, M., DARIO, P., "The Development of a Novel Prosthetic Hand-Ongoing Research and Preliminary Results", IEEE/ASME Transactions on Mechatronics, v. 7, 2006.

[11] SEBAStiAnI, F., ROCCELlA, S., VECCHI, F., CAROZZA, M.C., DARIO, P., "Experimental Analysis and Performance Comparison of three Different Prosthetic hands Designed According to a Biomechatronic Approach”, In: Proceedings of the 2003 IEEE/ASME International Conference on Advanced Intelligent Mechatronics., 2003.

[12] MIYATA, N., KOUCHI, M., KURIHARA, T., MOCHIMARU, M., "Modeling of Human Hand Link Sructure from Optical Motion Capture Data", Proceedings of 2004 IEEElRSJ International Conference on Intelligent Robots and Systems, 2004.

[13] RASH, G.S., BELLIAPPA, P.P., WACHOWIAK, M.P., SOMIA, N.N., GRUPTA, A., “A demonstration of the validity of a 3-D video motion analysis method for measuring finger flexion and extension”, Jornal of Biomechanics, v. 32, pp. 1337-1341, 1999.

[14] MIYATA, N., KOUCHI, M., MOCHIMARU, M., "Finger Joint Kinematic from MR Images", In: IEEE/RSJ International Conference on Intelligent Robots and Systems, 2005.

[15] CURA, V.O., CUNHA, F.L., AGUIAR, M.L., CLIQUET JR, A., "Implementação das Equações Interfalangeanas no Dedo da Mão de São Carlos, uma Prótese Multifunção”, XVIII Congresso Brasileiro de Engenharia Biomédica, São Jose dos Campos, SP, pp. 238-241, 2002.

[16] BERNE, R.M., LEVY, M.N., "A modified Procedure for Determining Inulin Space”, Proceedins of the Society for Experimental Biology and Medicine, v. 77, pp. 582-585, 1951.

[17] WU, G., VAN DER HELM, F.C.T., MOHSEN MAKHSOUS, H.E.J., ROY, P.V., ANGLIN, C., NAGELS, J., KARDUNA, A.R., MCQUADE, K., WANG, X., WERNER, F.W., BUCHHOLZ, B., "ISB Recommendation on Definitions of Joint Coordinate Systems of Various Joints for the Reporting of Human Joint Motion-Part II: Shoulder, Elbow, Wrist and Hand”, Jornal of Biomechanics, v. 38, pp. 981-992, 2005. 